\title{
Reconstruction of Chest Wall after Extensive Chest Wall Necrosis Caused by Transcatheter Arterial Embolization of Bilateral Internal Mammary Arteries Injured by Cardiopulmonary Resuscitation-A Case Report
}

\author{
Ayako Kamitomo*, Minoru Hayashi, Ryohei Tokunaka, Yuki Yoshida, Sayo Tatsuta, Yoshie Sasaki \\ Department of Plastic, Reconstructive and Aesthetic Surgery, Japan Red Cross Maebashi Hospital, Maebashi-shi, Japan \\ Email: ${ }^{\star}$ rachelkamitomo@gmail.com
}

How to cite this paper: Kamitomo, A., Hayashi, M., Tokunaka, R., Yoshida, Y., Tatsuta, S. and Sasaki, Y. (2019) Reconstruction of Chest Wall after Extensive Chest Wall Necrosis Caused by Transcatheter Arterial Embolization of Bilateral Internal Mammary Arteries Injured by Cardiopulmonary Resuscitation-A Case Report. Modern Plastic Surgery, 9, 33-43. https://doi.org/10.4236/mps.2019.92005

Received: March 10, 2019

Accepted: April 27, 2019

Published: April 30, 2019

Copyright $\odot 2019$ by author(s) and Scientific Research Publishing Inc. This work is licensed under the Creative Commons Attribution International License (CC BY 4.0).

http://creativecommons.org/licenses/by/4.0/ (c) (i) Open Access

\begin{abstract}
Transcatheter Arterial Embolization (TAE) is known to be effective for controlling severe hemorrhage caused by iatrogenic or blunt trauma. Out of more than 100 cases of TAE performed in our hospital, we have treated some cases of skin or muscle necrosis that resulted from embolization of the main arteries. In this study, we report the case of a patient with significant chest wall necrosis after TAE of the bilateral internal mammary arteries (IMAs). A 66-year-old male was transported to our hospital for loss of consciousness while playing golf. Cardiopulmonary resuscitation (CPR) was performed for cardiac arrest, which resulted in several rib fractures and mediastinal hematoma due to bilateral mammary artery injuries. Immediate TAE embolization was performed because of continuous hemorrhage. He was referred to our department 16 days after embolization due to the presentation of chest wall necrosis. Heart, lungs and diaphragm were exposed after surgical debridement under systemic anesthesia. We performed several operations to reconstruct the anterior chest wall. His spontaneous respiration returned, and is now controlled with a tracheostomy tube. Complete epithelialization was achieved, and he was transferred to another hospital for further rehabilitation. To the best of our knowledge, this is the first report of chest wall necrosis resulting from TAE of IMAs. Arterial embolization can cause widespread necrosis of bone, muscle and skin. Although treatment required an extended period, we managed to reconstruct the chest wall with multidisciplinary strategies.
\end{abstract}




\section{Keywords}

Transcatheter Arterial Embolization, Chest Wall Reconstruction, Internal Mammary Arteries, Negative Pressure Wound Therapy, Cardiopulmonary Resuscitation

\section{Introduction}

Internal mammary artery (IMA) injuries caused by iatrogenic or blunt trauma of the chest are rare. There have been previous reports of transcatheter arterial embolization (TAE) performed for mediastinal hemorrhage caused by IMA injuries [1]. However, there have been no reports of either chest wall necrosis following TAE or an appropriate treatment strategy. We present here a challenging case of anterior chest wall necrosis following TAE for mediastinal hemorrhage caused by IMA injuries resulting from cardiopulmonary resuscitation (CPR). To the best of our knowledge, this is the first report of chest wall necrosis resulting from TAE of IMAs. Arterial embolization can cause widespread necrosis of bone, muscle and skin. Although treatment required an extended period, we managed to reconstruct the chest wall with multidisciplinary strategies.

\section{Clinical Situation}

A 66-year-old male presented loss of consciousness and cardiac arrest while playing golf. He was transferred to our hospital and CPR was performed in the ambulance. Ventricular fibrillation was confirmed after 2 applications of automated external defibrillation followed by pulseless electrical activity. Since he had been periodically followed as an outpatient after a history of septic cardiomyopathy and ventricular tachycardia, he was highly suspected of having acute myocardial infarction, and immediate percutaneous coronary intervention was performed by cardiologists. He was intubated and an intra-aorta balloon pump was started. Stenosis of the right coronary artery was confirmed without complete obstruction, and ventricular fibrillation was suspected to have been caused by myocardial ischemia. Body computed tomography after percutaneous coronary intervention showed mediastinal hematoma, and the fracture of several ribs and the sternum. Contrast computed tomography showed extravasation in the anterior mediastinum (Figure 1). Immediate TAE was performed by a radiologist. Extravasation from the bilateral IMAs was confirmed by aortography, and the bilateral IMAs were selectively embolized (Figure 2). Reevaluation by contrast computed tomography on the 5th day showed extravasation from the right IMA and a growing mediastinal hematoma. He underwent an additional TAE of the right IMA with successful hemostasis.

On the 16th day, he was referred to our department due to the presentation of extensive eschar on the anterior chest wall (Figure 3). Computed tomography 


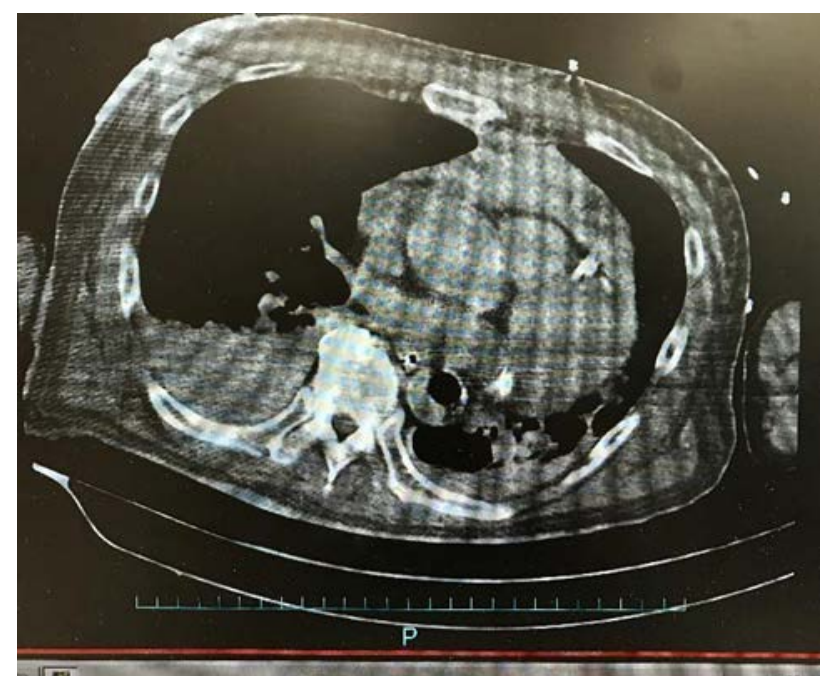

Figure 1. Computed tomography showing hemorrhage in thoracic cavity and mediastinum.

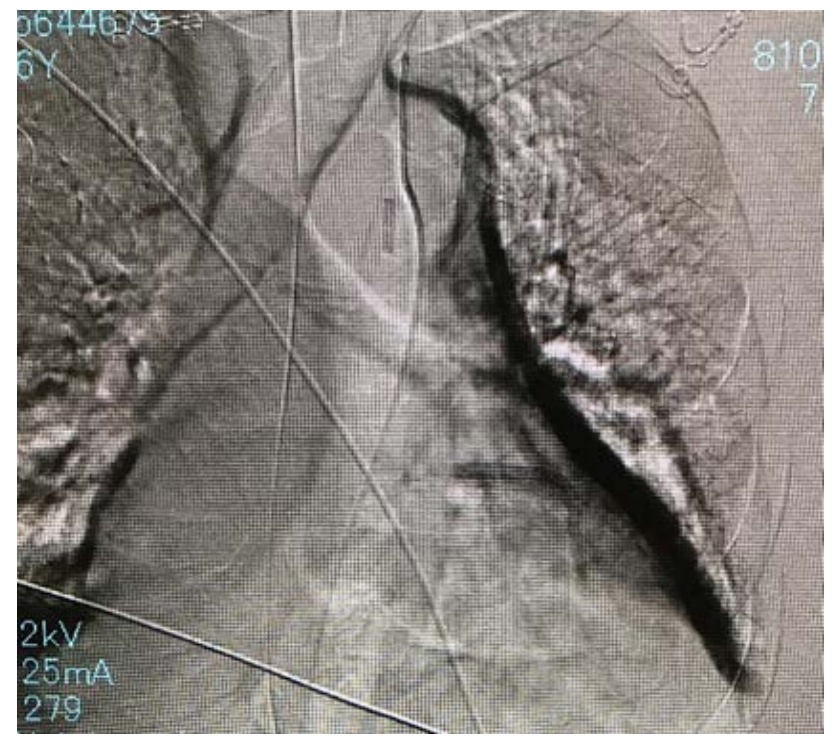

Figure 2. Angiography showing internal mammary artery. extravasation from bilateral internal mammary artery.

showed necrosis of the sternum, right first to third rib and left first to fourth rib (Figure 4).

\section{Methods}

We performed debridement of the anterior chest wall including the pectoralis major muscle, abdominal rectus muscle, internal intercostal muscle, part of the diaphragm, part of the right third rib and all the central ribs, and the left third rib and all the central ribs, which formed a defect that included the heart, lungs and diaphragm (Figure 5), to which we applied negative pressure wound therapy (NPWT) (Figure 6). Thoracic drainage tubes were placed bilaterally. We periodically exchanged the device. On the 56th day, we performed a second 


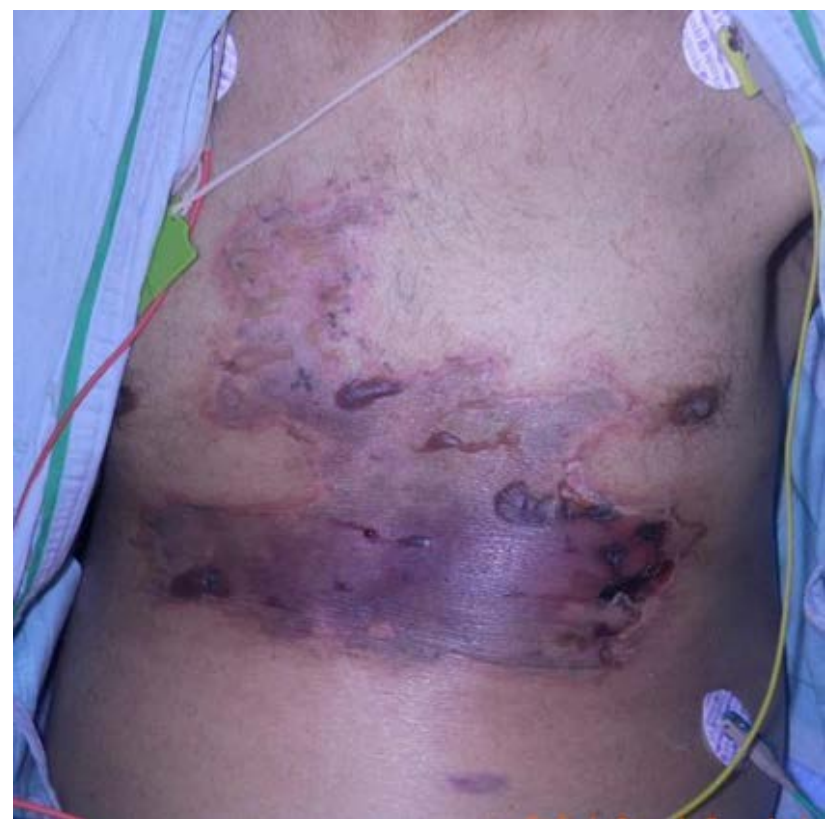

Figure 3. The chest wall necrosis after transcatheter arterial embolization.

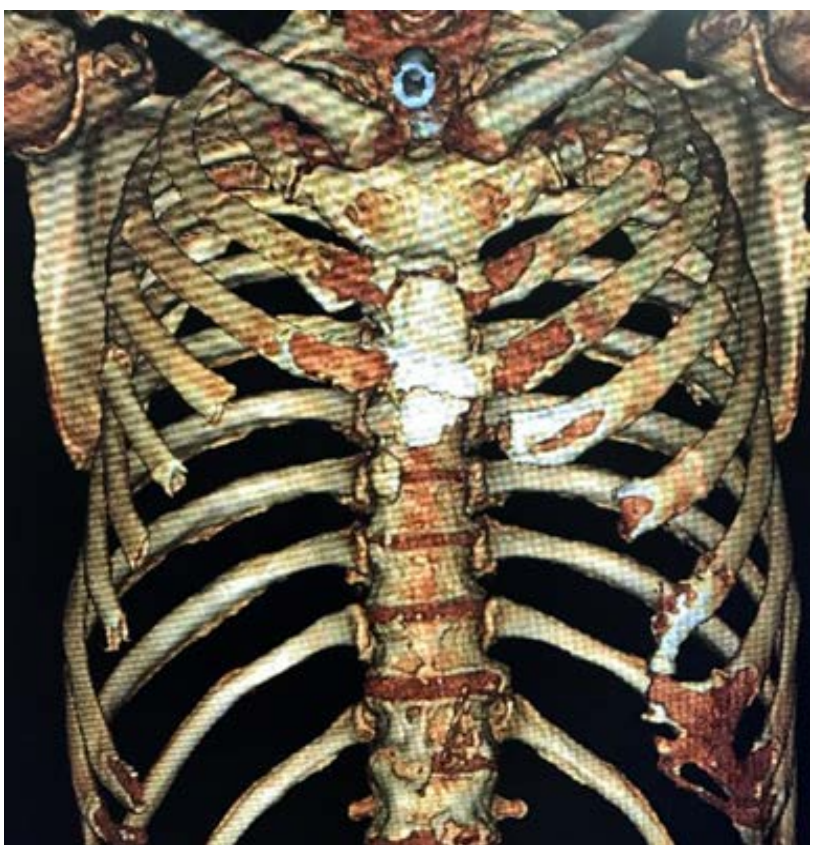

Figure 4. 3D computed tomography showing necrosis of right first to third rib and left first to fourth rib.

operation to reconstruct the chest wall using bilateral latissimus dorsi myocutaneous flaps with ribs. In the supine position, additional debridement was performed for the cartilage of the right third rib and the body of the sternum, which was complicated by the leakage of air from part of the right upper lung. In the right lateral position, the left latissimus dorsi flap was harvested without a skin paddle including the left ninth and tenth ribs without periosteum with intercostal 


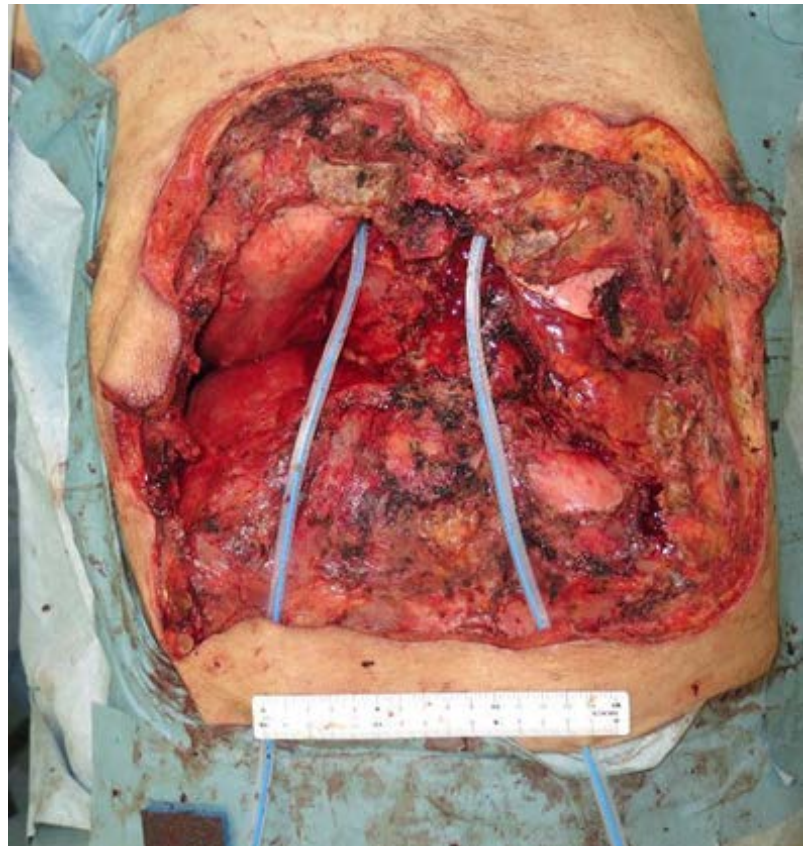

Figure 5. The exposed heart, lungs and diaphragm after the debridement.

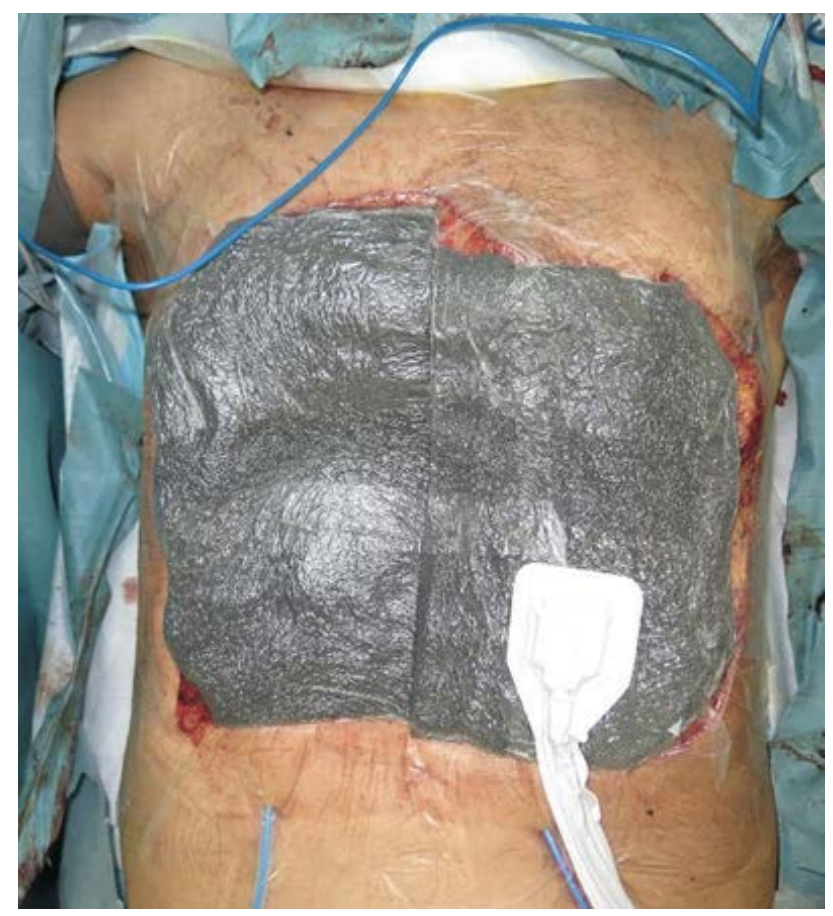

Figure 6. NPWT applied after the debridement.

muscle. The right latissimus dorsi flap was similarly harvested, which involved the tenth and eleventh ribs with a $70 \times 220 \mathrm{~mm}$ skin paddle. Both donor sites were closed with insertion of a drainage tube. Both flaps were transpositioned into the defect through a lateral thoracic tunnel. The sternum segment and the harvested left ribs with a skin paddle were fixed with titanium wire, as well as the 
harvested ninth rib with a union segment of the sixth and seventh ribs. The harvested tenth rib was sutured. A split-thickness skin graft was harvested from the right thigh and placed on the rest of the chest wall (Figure 7), to which we applied a NPWT device.

A week after the operation, bilateral distal flaps were found to be necrotic and infected. They were soon debrided, which led to the reappearance of ribs with confirmation of air leakage. Since he had a medical history of calciphylaxis, he suffered poor wound healing.

On the 85th day, we planned another reconstruction operation using an anterolateral thigh free flap. We chose this flap for its coverage of the middle part of chest which protects the heart and exposed ribs. The flap was harvested from the left thigh and included the majority of the vastus lateralis muscle along with the descending branch of the circumflex femoral artery and 3 perforator vessels. Through a zigzag incision on the left neck, the left superior thyroid vein and communicating vein were identified. The concomitant vein was anastomosed to the left superior thyroid vein and communicating vein. The muscle flap was transpositioned beneath the right grafted ribs to eliminate air leakage (Figure 8). A NPWT device was placed on artificial dermis that was applied to the rest of the defect. The flap survived with the successful elimination of air leakage from the right upper lung (Figure 9). An additional skin graft operation was planned on the 127th day.

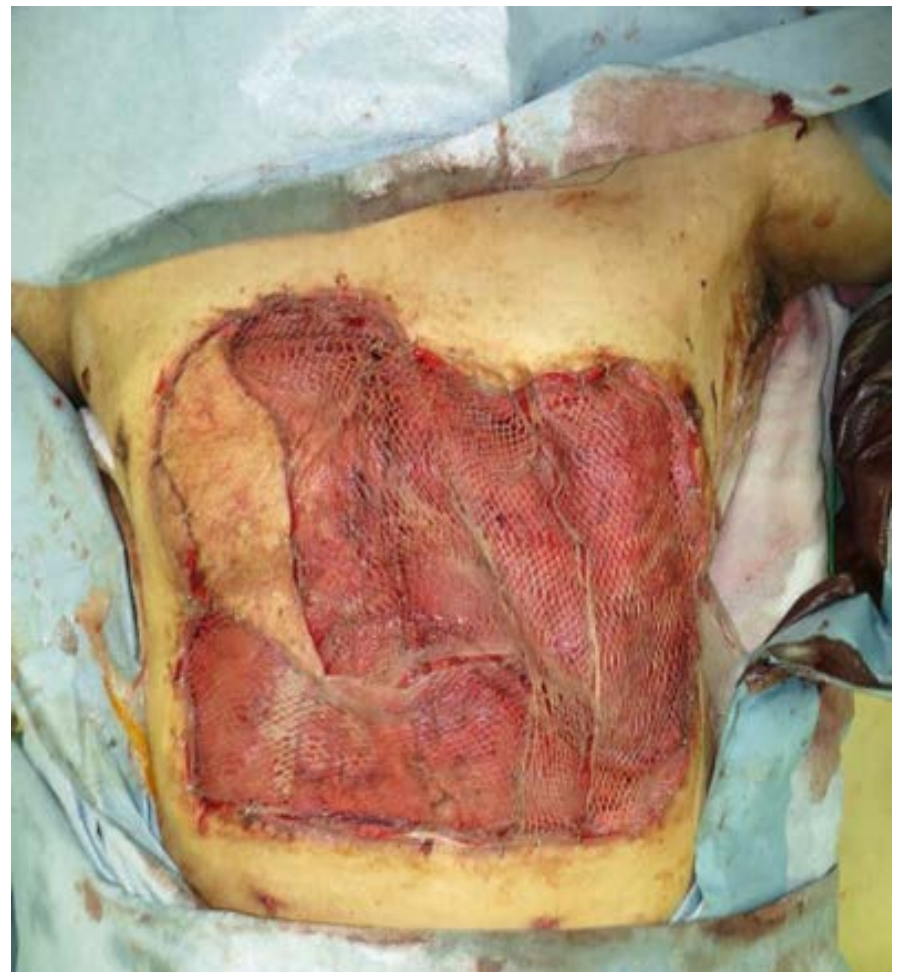

Figure 7. Immediate result after the reconstruction using bilateral latissimus dorsimyocutaneous flap with the ribs and a split-thickness skin graft. 


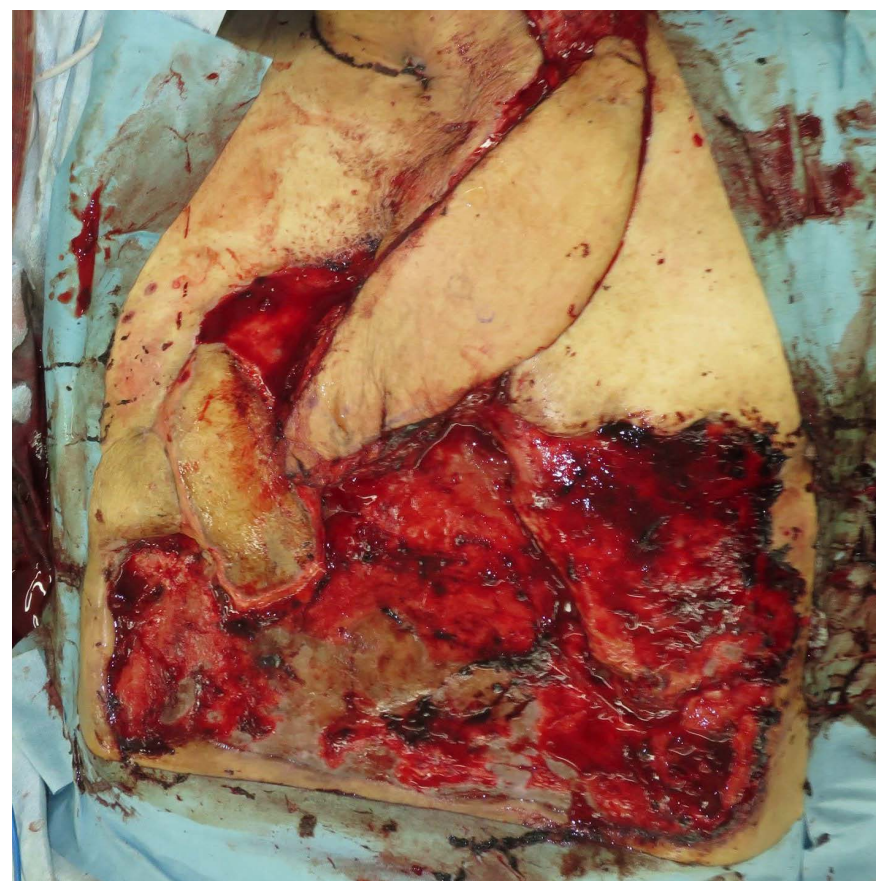

Figure 8. Immediate result after the reconstruction using anterior lateral thigh flap.

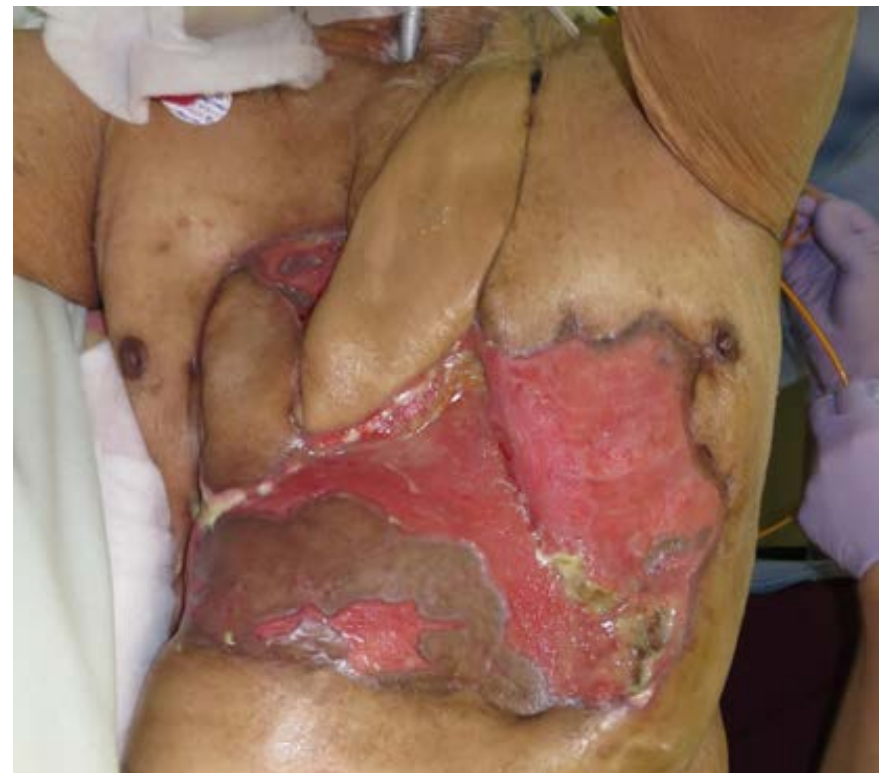

Figure 9. The anterior lateral thigh flap survived with successful elimination of air leakage.

\section{Results}

Ultimately, complete coverage of the chest wall was achieved on the 175th day. His respiration is now managed with a tracheostomy tube with spontaneous respiration (Figure 10). Nonverbal communication is possible. He was transferred to another hospital for further continuous rehabilitation. No complication have been confirmed for further follow-up. 


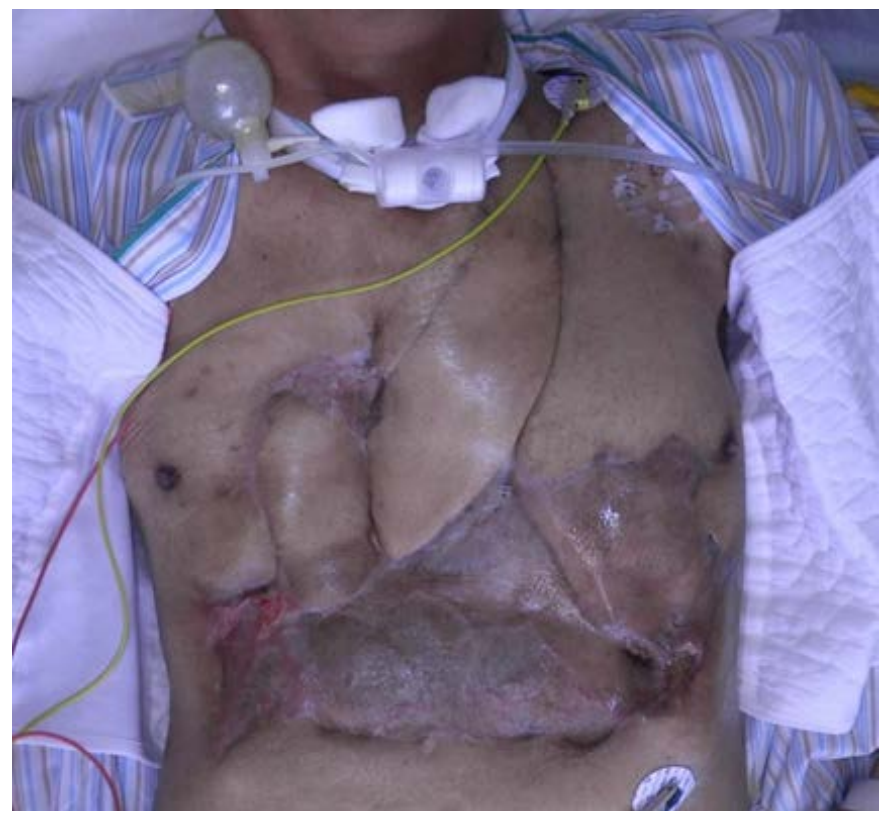

Figure 10. Complete epithelialization obtained on 175th day. A tracheostomy tube was inserted.

\section{Discussion}

IMA injuries can occur after blunt trauma or be iatrogenic. They often accompany rib or sternum fracture. Continuous bleeding can lead to the development of mediastinal hematoma or hemothorax, which should be immediately controlled. Whereas IMA injuries have mainly been due to blunt trauma, a growing number of cases are caused by CPR, for which the recommended compression depth has been increased to $5 \mathrm{~cm}$ [2]. Kawakami et al. reported a case of IMA injury related to CPR with successful TAE [3]. Yamagishi et al. reported five cases of IMA injuries related to CPR with TAE followed by no complications [4]. TAE is a reliable treatment option for IMA injuries and some cases have been reported despite a controversy regarding whether to choose thoracotomy or conservative embolization [5]. Our patient underwent TAE of the bilateral IMAs, which was complicated by the formation of an ischemic skin change on the chest wall 17 days after TAE. To the best of our knowledge, this is the first reported case of extensive chest wall necrosis after TAE of IMA. Whigham et al. reported that 12 of 18 cases of hemorrhage from an intercostal artery were successfully treated with TAE without any complications [6]. Chemelli et al reported that there were no necrotic complications of the chest after TAE for intercostal artery injuries [7]. Corvino et al. also reported no major or minor complications after TAE of IMA [8]. Since there are no previous reports of chest wall necrosis, the incidence of this complication is unknown.

IMA is the third branch of the subclavian artery. It descends peripherally and bifurcates into the musculophrenic artery and superior epigastric artery. Extensive necrosis of the chest wall, flail chest or malfunction of the diaphragm can be considered a complication of TAE of IMA. When our patient was referred to our 
department, he had ischemic skin change with a clear margin, which was extensively debrided during the operation to prevent the outbreak and progression of sepsis. The defect after debridement included the heart, lungs, some ribs and diaphragm, and we applied an NPWT device to promote the formation of granulation to be covered with tissue. Reconstruction of the chest wall was challenging in several respects. There have been previous reports on chest wall reconstruction and various strategies [9] [10]. In terms of chest wall defects, treatment with the use of a vacuum-assisted closure device for sternal osteomyelitis for surgical site infection has been widely reported. However, there have been no reports in which an exposed heart was treated with NPWT, as in our patient. We placed non-adherent mesh between polyurethane foam and the heart to prevent adhesion. Since the chest wall protects the intrathoracic organs and respiratory function, extensive defects may lead to a loss of chest wall stability and paradoxical movement of the lungs. Reconstruction should be planned from a multidisciplinary point of view, and should involve both a thoracic surgeon and a plastic surgeon. Knowledge of the chest wall anatomy is essential. In this case, the goal of reconstructing the chest wall was to restore stability and integrity, protect the intrathoracic organs, repair the air leak from the lung and move him off the ventilator. We first needed to plan how to achieve skeletal stability after a significant loss of ribs. We chose bilateral latissimus dorsi myocutaneous flaps with ribs, which were fixed with sternum wire to recipient ribs. After achieving skeletal rigidity, coverage by soft tissue was necessary. We chose an anterolateral thigh flap to cover the chest wall and repair the air leak. Due to his medical history of calciphylaxis, significant calcification of blood vessels was found, which made blood distribution difficult and resulted in necrosis of the bilateral distal latissimus dorsi flaps. He also showed poor wound healing of the skin graft. We eventually were able to move him off the ventilator support with the elimination of air leakage, and his respiration is currently being controlled by only a tracheostomy tube. On the 233rd day, he was referred to another hospital for further rehabilitation.

There are several possible explanations for what triggered necrosis of the chest wall. Previous reports have examined what contributes to the formation of necrotic gluteal skin changes after TAE of internal iliac artery after hemorrhage caused by pelvic fracture. Yamamoto reported that skin and muscle necrosis after TAE can be attributed to the damage sustained at the time of the accident rather than to TAE itself [11]. Suzuki reported that skin and muscle necrosis are aggravated by the combination of decreased blood flow due to embolization and the ischemia of soft tissue caused by the injury [12]. Yoshino reported that advances in technology have contributed to the improved performance of TAE of IMA sparing its main trunk. He also stated that, regarding TAE of IMA, there has been little discussion of the importance of preserving its main trunk by selective arterial embolization through the use of advanced technologies, considering the possible complications caused by nonselective embolization, such as the overflow of the embolization material toward a vertebral artery or the out- 
flow of chemo-drugs to the skin [13]. While there has been no statistical analysis of what causes chest wall necrosis after TAE of IMA, we think that, in our patient, the damage to the anterior chest wall caused by CPR and his medical history of calciphylaxis increased the risk of complications.

\section{Conclusion}

TAE is minimally invasive and therefore favorable for controlling mediastinal hemorrhage due to internal artery injuries. However, during the course of treatment, we need to consider the possible serious complications associated with obstructions of IMAs, such as chest wall necrosis. Since the 2010 American Heart Association Guidelines emphasize the need for CPR and recommend compression of more than $5 \mathrm{~cm}$, the number of CPR-related IMA injuries may increase. Early diagnosis followed by immediate debridement needs to be performed to prevent infective complications. This is the first reported case of chest wall reconstruction after extensive chest wall necrosis caused by TAE of bilateral IMAs injured by CPR, which was successfully treated with multidisciplinary planning.

\section{Consent}

The patient's family had given their consent for the case report to be published.

\section{Conflicts of Interest}

The authors declare no conflicts of interest regarding the publication of this paper.

\section{Financial Disclosure}

None of the authors has a financial interest in any of the products, devices, or drugs mentioned in this manuscript.

\section{References}

[1] Kawamura, S., Nishimaki, H., Takigawa, M., et al. (2006) Internal Mammary Artery Injury after Blunt Chest Trauma Treated with Transcatheter Arterial Embolization. Journal of Trauma, 61, 1536-1539. https://doi.org/10.1097/01.ta.0000243201.49744.fc

[2] Hallevuo, H., Sainio, M., Nevelainen, R., et al. (2013) Deeper Chest CompressionMore Complications for Cardiac Arrest Patients? Resuscitation, 84, 760-765. https://doi.org/10.1016/j.resuscitation.2013.02.015

[3] Kawakami, S., Noguchi, T., Doi, T., et al. (2016) Internal Mammary Artery Injury Related to Chest Compressions in a Patient with Post-Cardiac Arrest Syndrome. Internal Medicine, 55, 1299-1303. https://doi.org/10.2169/internalmedicine.55.5762

[4] Yamagishi, T., Kashiura, M., Sugiyama, M., et al. (2017) Chest Compression-Related Fatal Internal Mammary Artery Injuries Manifesting after Venoarterial Extracorporeal Membrane Oxygenation: A Case Series. Journal of Medical Case Reports, 11, 318. https://doi.org/10.1186/s13256-017-1485-y

[5] Nonomi, H., Ootsuka, T., Horio, H., et al. (2003) Bilateral Internal Thoracic Artery 
Injury Induced by Blunt Trauma. The Journal of Thoracic and Cardiovascular Surgery, 51, 214-216. https://doi.org/10.1007/s11748-003-0036-1

[6] Whigham, C.J., Fisher, R.G., Goodman, C.J., et al. (2002) Traumatic Injury of the Internal Mammary Artery: Embolization versus Surgical and Nonoperative Management. Emergency Radiology, 9, 201-207.

[7] Chemelli, A.P., Thauerer, M., Wiedernann, F., et al. (2009) Transcatheter Arterial Embolization for the Management of Iatrogenic and Blunt Traumatic Intercostal Artery Injuries. Journal of Vascular Surgery, 49, 1505-1513.

https://doi.org/10.1016/j.jvs.2009.02.001

[8] Corvino, F., Giurazza, F., Cangiano, G., et al. (2018) Safety and Effectiveness of Transcatheter Embolization in the Treatment of Internal Mammary Artery Injuries. Radiology Medicine, 123, 369-377. https://doi.org/10.1007/s11547-017-0844-5

[9] Mahabir, R.C. and Butler, C.E. (2011) Stabilization of the Chest Wall: Autologous and Alloplastic Reconstructions. Seminars in Plastic Surgery, 25, 34-42. https://doi.org/10.1055/s-0031-1275169

[10] Christopher, S. and Gaetano, R. (2016) Chest Wall Reconstruction after Extended Resection. Journal of Thoracic Disease, 8, 863-871. https://doi.org/10.21037/jtd.2016.11.07

[11] Yamamoto, R., Imai, S., Gyouten, M., et al. (2002) Usefulness and Complications of Transcatheter Arterial Embolization for the Treatment of Pelvic Fracture; Evaluation of 27 Cases. Japanese Journal of Clinical Radiology, 47, 1157-1161.

[12] Suzuki, T., Shindo, M., Kataoka, Y., et al. (2005) Clinical Characteristics of Pelvic Fracture Patients with Gluteal Necrosis Resulting from Transcatheter Arterial Embolization. Archives of Orthopaedic and Trauma Surgery, 125, 448-452. https://doi.org/10.1007/s00402-005-0827-1

[13] Yoshino, Y., Yamada, Y., et al. (2017) Recent Advances in Embolization for Internal Mammary Artery. Journal of the Japanese Society for Endovascular Intervention, 18, 14-19. 